\title{
Observing Pose and Motion through Contact*
}

\author{
Yan-Bin Jia Michael Erdmann \\ The Robotics Institute \\ School of Computer Science \\ Carnegie Mellon University \\ Pittsburgh, PA 15213-3890
}

\begin{abstract}
This paper investigates how to "observe" a planar object being pushed by a finger. The pushing is governed by a nonlinear system that relates through contact the object pose and motion to the finger motion. Nonlinear observability theory is employed to show that the contact information is often sufficient for the finger to determine not only the pose but also the motion of the object. Therefore a sensing strategy can be realized as an observer of the nonlinear dynamical system, which is subsequently introduced. The observer, based on the result of [6], has its "gain" determined by the solution of a Lyapunov-like equation.

Simulations have been done to demonstrate the feasibility of the observer. A sensor has been implemented using strain gauges and mounted on an Adept robot with which preliminary experiments have been conducted.

From a general perspective, this work presents an approach for acquiring geometric and dynamical information about a task from a small amount of tactile data, with the application of nonlinear observability theory.
\end{abstract}

\section{Introduction}

Sensing and grasping are often performed sequentially by robots. But this is not the case with human beings. Even with no help from vision, the human hand can usually manipulate an object by feeling the contact and utilizing this information to control the object.

For example, try to grasp something, say, a pen, on the table while keeping your eyes closed. Your hand gropes for it on the table until one of the fingers touches the pen and starts pushing it for a short distance. By feeling if the contact is almost stable, moving counterclockwise, or moving clockwise on the fingertip, you can quickly tell

\footnotetext{
* Support for this research was provided in part by Carnegie Mellon University, and in part by the National Science Foundation through the following grants: NSF Presidential Young Investigator award IRI-9157643 and NSF Grants IRI-9213993 and IRI-9503648.
}

if the middle, the right end, or the left end of the pen is being touched, respectively. Immediately, you are able to coordinate other fingers to close in for a grip.

The above example suggests that the human hand has some intrinsic way of exploiting the shape information about an object and the tactile information generated by a mechanical interaction with the object. In designing a sensing or grasping strategy, we should try to take advantage of the mechanics, the manipulator and object's geometry, and their correlation. This paper illustrates such idea through the study of a specific problem: Can we determine the pose and motion of a known object from the contact motion on a pushing finger?

In this paper we will answer yes in general to the above question, offering a pose and motion estimation algorithm in Section 4. Figure 1 shows a simple example of a disk pushing a 7-gon and estimating the pose and motion of the polygon in less than a second using the algorithm.

\subsection{Related Work}

Dynamics of sliding rigid bodies was treated by MacMillan [15] for non-uniform pressure distributions, and by Goyal et al. [7] using geometric methods based on the limit surface description of friction.

Montana [17] derived a set of differential equations describing the motion of a contact point in response to a relative motion of the objects in contact. The kinematics of spatial motion with point contact was also studied by Cai and Roth [3].

Mason [16] pioneered the study of the mechanics of pushing using quasi-static analysis. Alexander and Maddocks [1] offered analytical solution to the problem of determining the motion of a slider under applied force by a reduction to the case of a bipod. Lynch et al. [14] localized an object using the mechanics of pushing and tactile feedback.

The paper by Salisbury [19] introduced fingertip force sensing which determines contact locations and orientations from force and moment measurements. Fearing and Bin- 


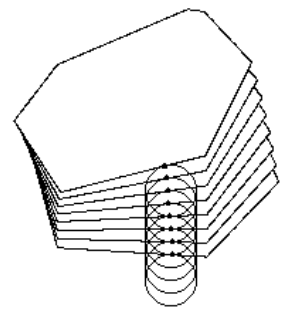

(a) Actual Scene

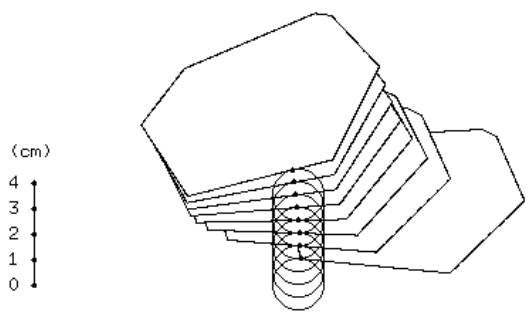

(b) Perceived Scene
Figure 1: A disk of radius $1 \mathrm{~cm}$ at constant velocity $5 \mathrm{~cm} / \mathrm{s}$ pushing a 7-gon $\mathcal{P}$ while observing its pose and motion. Contact friction is large enough to allow only the rolling of the 7-gon on the disk edge. The coefficient of support friction is 0.3. (a) The scene of pushing for $0.71 \mathrm{~s}$. (b) The imaginary scene as "perceived" by the observer during the same time period. Although the real contact and its estimate were about $4.5 \mathrm{~cm}$ apart on the contact edge at the start of estimation, the error becomes negligible in $0.56 \mathrm{~s}$.

ford [5] designed a cylindrical tactile sensor to determine the principal curvatures of an object through rolling contact. Inspired by the results on exploratory procedures in human haptics, Allen and Roberts [2] fit a number of contact points around an object obtained by robot fingers to a superquadric surface representation to reconstruct the object's shape.

The foundation of our work comes from the theory of the observability and observers of nonlinear systems. For a general introduction to nonlinear control theory, we refer the reader to Isidori [9] and Nijmeijer and van der Schaft [18].

Necessary and sufficient conditions for linearization by output injection for autonomous nonlinear systems (i.e., without input) were given in [11] by Krener and Isidori, and in [12] by Krener and Respondek along with a constructive algorithm.

Gauthier, Hammouri, and Othman [6] described an observer for affine-control nonlinear systems whose "gain" is determined via the solution of an appropriate Lyapunovlike equation. Ciccarella et al. [4] proposed a similar observer whose gain vector is controlled by the properly chosen eigenvalues of a certain matrix obtained from the original system's Brunowsky canonical form.

Zimmer [20] presented a state estimator based on Newton's method that conducts on-line minimization over some objective function.

\section{Motion of Contact}

Throughout the paper we consider the two-dimensional problem of a translating finger $\mathcal{F}$ pushing an object $\mathcal{B}$.
The coefficient of support friction between $\mathcal{B}$ and the plane is everywhere $\mu$. Object $\mathcal{B}$ has uniform mass and pressure distributions. Let us assume frictionless contact between $\mathcal{F}$ and $\mathcal{B}$ at present and discuss contact friction in Section 5. Let $\boldsymbol{v}_{\mathcal{F}}$ be the velocity of $\mathcal{F}$, known to $\mathcal{F}$ 's controller, $\boldsymbol{v}$ and $\omega$ the velocity and angular velocity of $\mathcal{B}$, respectively, all in the world coordinate frame (Figure 2).

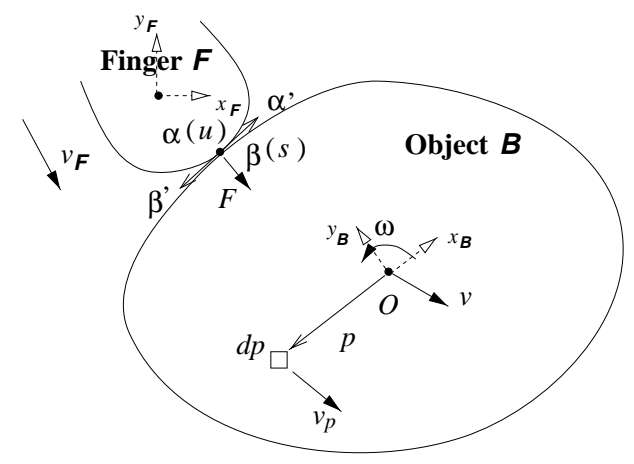

Figure 2: Finger $\mathcal{F}$ translating and pushing object $\mathcal{B}$

Let $\mathcal{F}$ be bounded by a smooth curve $\alpha$ and $\mathcal{B}$ bounded by a piecewise smooth closed curve $\beta$ such that $\alpha(u)$ and $\beta(s)$ are the two points in contact in the local frames of $\mathcal{F}$ and $\mathcal{B}$, respectively. Without loss of generality, $\alpha$ and $\beta$ are unitspeed curves with curvatures $\kappa_{\alpha}$ and $\kappa_{\beta}$ (where defined), respectively. Assume that one curve segment of $\beta$ stays in contact with $\alpha$ throughout the pushing and that $\alpha$ and $\beta$ are always convex in small neighborhoods of the two contact points. To avoid any ambiguity, the notation ". ' means differentiation with respect to time, while the notation ", means differentiation with respect to some curve parameter. For example, $\dot{\alpha}=\alpha^{\prime} \dot{u}=\frac{d \alpha}{d u} \frac{d u}{d t}$ gives the velocity of the contact point on the fingertip.

That $\mathcal{F}$ and $\mathcal{B}$ maintain contact imposes a velocity constraint:

$$
\boldsymbol{v}_{\mathcal{F}}+\alpha^{\prime} \dot{u}=\boldsymbol{v}+\omega \times R \beta+R \beta^{\prime} \dot{s},
$$

where $R(\theta)$ is the rotation matrix associated with the orientation $\theta$ of $\mathcal{B}$, which is determined by the orientation of $\mathcal{F}$, $u$, and $s$. In our previous work [10], we derived the contact and object motions from (1), the geometric constraints of contact, and Newton's and Euler's equations for dynamics.

Theorem 1 In the given system of $\mathcal{F}$ pushing $\mathcal{B}$, the points of contact evolve according to

$$
\begin{aligned}
& \dot{u}=\frac{\omega+\kappa_{\beta} \alpha^{\prime} \cdot\left(\boldsymbol{v}+\omega \times R \beta-\boldsymbol{v}_{\mathcal{F}}\right)}{\kappa_{\alpha}+\kappa_{\beta}} ; \\
& \dot{s}=\frac{-\omega+\kappa_{\alpha} \alpha^{\prime} \cdot\left(\boldsymbol{v}+\omega \times R \beta-\boldsymbol{v}_{\mathcal{F}}\right)}{\kappa_{\alpha}+\kappa_{\beta}}
\end{aligned}
$$


and the object's angular acceleration and acceleration are given as

$$
\begin{aligned}
\dot{\omega}= & -\left(\dot{u} \alpha^{\prime \prime} \times\left(\boldsymbol{v}_{\mathcal{F}}-\boldsymbol{v}\right)-\left(\dot{u} \alpha^{\prime \prime} \cdot R \beta+\left(\beta^{\prime} \times \beta\right) \omega-\dot{s}\right) \omega\right. \\
& \left.+\alpha^{\prime} \times \boldsymbol{a}_{\mathcal{F}}+\frac{\mu g}{\mathcal{A} \beta^{\prime} \cdot \beta} \alpha^{\prime} \times \Gamma\right) /\left(\beta^{\prime} \cdot \beta+\frac{\rho^{2}}{\beta^{\prime} \cdot \beta}\right),(4) \\
\dot{\boldsymbol{v}}= & \frac{\mathcal{A} \rho^{2} \dot{\omega} \times R \beta^{\prime}-\mu g \Gamma}{\mathcal{A} \beta^{\prime} \cdot \beta},
\end{aligned}
$$

where $\boldsymbol{a}_{\mathcal{F}}$ is the acceleration of $\mathcal{F}, \mathcal{A}$ and $\rho$ the area and radius of gyration of $\mathcal{B}$, respectively, $g$ the acceleration of gravity. The integral $\Gamma=\int_{\mathcal{B}} R \beta^{\prime} \times\left(R \boldsymbol{p} \times \hat{\boldsymbol{v}}_{p}\right)+\left(\beta^{\prime}\right.$. $\beta) \hat{\boldsymbol{v}}_{p} d \boldsymbol{p}$ is associated with support friction. Here $\boldsymbol{v}_{p}=$ $\boldsymbol{v}+\omega \times R \boldsymbol{p}$ is the velocity of $\boldsymbol{p} \in B$ and $\hat{\boldsymbol{v}}_{p}=\frac{v_{p}}{\left|v_{p}\right|}$ its direction. $^{1}$

\section{Local Observability}

Given the nonlinear system (2)-(5), sensing can be viewed as to determine the object contact $s$, and possibly, the object's angular velocity $\omega$ and velocity $v$, from the finger contact $u$. In this section we shall study whether $u$ contains enough information for such computation, resorting to the notion of local observability in nonlinear control theory.

\subsection{Nonlinear Local Observability}

Let us consider a smooth affine (or input-linear) control system together with an output map:

$\dot{\boldsymbol{x}}=\boldsymbol{f}(\boldsymbol{x})+\sum_{i=1}^{m} u_{i} \boldsymbol{g}_{i}(\boldsymbol{x}), \boldsymbol{u}=\left(u_{1}, \ldots, u_{m}\right) \in U \subset \Re^{m}$,

$\boldsymbol{y}=\boldsymbol{h}(\boldsymbol{x})$,

where $\boldsymbol{x}=\left(x_{1}, \ldots, x_{n}\right)^{T}$ is the state in a smooth $n$ dimensional manifold $M \subseteq \Re^{n}$ (called the state space manifold), $\boldsymbol{f}, \boldsymbol{g}_{1}, \ldots, g_{m}$ are smooth vector fields on $M$, and $\boldsymbol{h}=\left(h_{1}, \ldots, h_{k}\right)^{T}: M \rightarrow \Re^{k}$ is the smooth output map of the system. Here $f$ is called the drift vector field and $g_{1}, \ldots, g_{m}$ the input vector fields. In the system, $u_{1}, \ldots, u_{m}$ are the inputs, called the controls. Throughout we are only concerned with the class $\mathcal{U}$ of admissible controls that are piecewise constant functions and continuous from the right.

Denote by $\boldsymbol{y}\left(t, \boldsymbol{x}_{0}, \boldsymbol{u}\right), t \geq 0$, the output function of the system with initial state $\boldsymbol{x}_{0}$ and under control $\boldsymbol{u}$. Two

\footnotetext{
${ }^{1}$ Equations (2)-(5) are numerically solvable for $u, s, v$, and $\omega$. Closed forms of $\Gamma$ exist for polygonal objects; for most other shapes, it has to be evaluated numerically. At the initial state, the object and the finger are motionless. The initial accelerations are solvable using Newton's method [10].
}

states $\boldsymbol{x}_{1}, \boldsymbol{x}_{2} \in M$ in an open set $V \subset M$ are said to be $V$-indistinguishable, denoted $\boldsymbol{x}_{1} I^{V} \boldsymbol{x}_{2}$, if for any $T>0$ and any constant control $\boldsymbol{u}:[0, T] \rightarrow U$ such that $\boldsymbol{x}\left(t, \boldsymbol{x}_{1}, \boldsymbol{u}\right), \boldsymbol{x}\left(t, \boldsymbol{x}_{2}, \boldsymbol{u}\right) \in V$ for all $0 \leq t \leq T$, it follows that $\boldsymbol{y}\left(t, \boldsymbol{x}_{1}, \boldsymbol{u}\right)=\boldsymbol{y}\left(t, \boldsymbol{x}_{2}, \boldsymbol{u}\right)$ for all $0 \leq t \leq T$. The system is locally observable at $\boldsymbol{x}_{0}$ if there exists a neighborhood $W$ of $\boldsymbol{x}_{0}$ such that in every neighborhood $V \subset W$ of $\boldsymbol{x}_{0}$ the relation $\boldsymbol{x}_{0} I^{V} \boldsymbol{x}_{1}$ implies that $\boldsymbol{x}_{0}=\boldsymbol{x}_{1}$. The system is called locally observable if it is locally observable at every $\boldsymbol{x}_{0} \in M$.

The Lie derivative of function $h_{j}: M \rightarrow \Re$ along a vector field $X$ on $M$, denoted by $L_{X} h_{j}$, is the directional derivative $d h_{j}(X)=d h_{j} \cdot X$, where $d h_{j}=\left(\frac{\partial h_{j}}{\partial x_{1}}, \ldots, \frac{\partial h_{j}}{\partial x_{n}}\right)$ is the differential or gradient of $h$. The observation space $\mathcal{O}$ of system (6) is the linear space (over $\Re$ ) of functions on $M$ that includes $h_{1}, \ldots, h_{k}$, and all repeated Lie derivatives:

$$
L_{X_{1}} L_{X_{2}} \cdots L_{X_{l}} h_{j}=L_{X_{1}}\left(L_{X_{2}}\left(\ldots\left(L_{X_{l}} h_{j}\right) \ldots\right)\right) \text {, }
$$

for $j=1, \ldots, k, \quad l=1,2, \ldots$, and $X_{1}, \ldots, X_{l} \in$ $\left\{\boldsymbol{f}, \boldsymbol{g}_{1}, \ldots, \boldsymbol{g}_{m}\right\}$. The observability codistribution at state $\boldsymbol{x} \in M$, denoted $d \mathcal{O}(\boldsymbol{x})$, is defined as:

$$
d \mathcal{O}(\boldsymbol{x})=\operatorname{span}\{d H(\boldsymbol{x}) \mid H \in \mathcal{O}\}
$$

We refer the reader to [8] and [18, pp. 95-96] for more on nonlinear observability.

Theorem 2 (Herman and Krener) System (6) is locally observable at state $\boldsymbol{x}_{0} \in M$ if $\operatorname{dim} d \mathcal{O}\left(\boldsymbol{x}_{0}\right)=n$.

The equation $\operatorname{dim} d \mathcal{O}\left(\boldsymbol{x}_{0}\right)=n$ is called the observability rank condition. Basically, to distinguish between a state and any other state in its neighborhood, it is necessary to consider not only the output functions but also their derivatives along all possible system trajectories. The rank condition ensures the existence of $n$ output functions and/or derivatives which together define a diffeomorphism on some neighborhood of the state, which in turn ensures that the state is locally distinguishable.

\subsection{The Disk-Polygon System}

Now we study the case of pushing in which finger $\mathcal{F}$ is a disk bounded by $\alpha=r\left(\cos \frac{u}{r}, \sin \frac{u}{r}\right)^{T}$ and object $\mathcal{B}$ is a simple polygon. This type of pushing is representative of real tasks. The interior of one edge $e$ of $\mathcal{B}$ is in contact with $\mathcal{F}$ throughout the pushing. Since a sensing strategy can hypothesize all edges of $\mathcal{B}$ as the contact edge and verify them one by one, we assume that $e$ is known. Let $h$ be the distance from the centroid $O$ of $\mathcal{B}$ to $e$. Choose $s$ as the signed distance from the contact to the intersection of $e$ and its perpendicular through $O$ such that $s$ increases while moving counterclockwise on $e$, as shown in Figure 3. The 


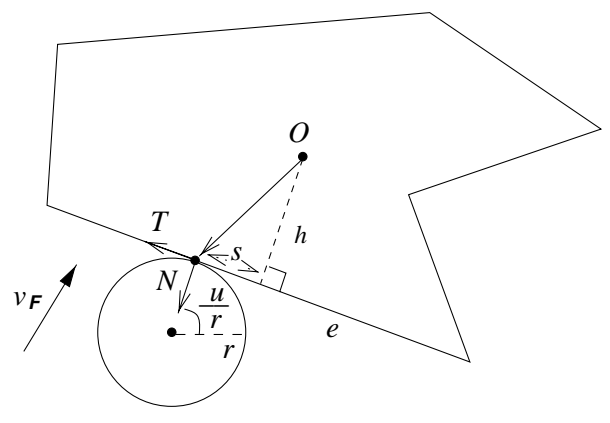

Figure 3: A circular finger pushing a polygonal object.

orientation of $\mathcal{B}$ is $\theta=u / r-\pi / 2 .^{2}$ The tangent and normal of $\mathcal{F}$ at the contact are $T=\alpha^{\prime}=\left(-\sin \frac{u}{r}, \cos \frac{u}{r}\right)^{T}$ and $N=r \alpha^{\prime \prime}=-\left(\cos \frac{u}{r}, \sin \frac{u}{r}\right)^{T}$, respectively. The system (2)(5) now reduces to ${ }^{3}$

$$
\begin{aligned}
\dot{u} & =\omega r ; \\
\dot{s} & =T \cdot\left(\boldsymbol{v}-\boldsymbol{v}_{\mathcal{F}}\right)-\omega(r+h) ; \\
\dot{\omega}= & \frac{s}{s^{2}+\rho^{2}}\left(\omega^{2}(r+h)-2 \omega T \cdot\left(\boldsymbol{v}-\boldsymbol{v}_{\mathcal{F}}\right)-T \times \boldsymbol{a}_{\mathcal{F}}\right) \\
& \quad-\frac{\mu g}{\mathcal{A}\left(s^{2}+\rho^{2}\right)} T \times \Gamma ; \\
\dot{\boldsymbol{v}}= & \frac{\rho^{2}}{s^{2}+\rho^{2}}\left(N \cdot \boldsymbol{a}_{\mathcal{F}}-\omega^{2}(r+h)+2 \omega T \cdot\left(\boldsymbol{v}-\boldsymbol{v}_{\mathcal{F}}\right)\right) N \\
& \quad-\frac{\mu g}{\mathcal{A} s}(T \cdot \Gamma) T-\frac{s}{s^{2}+\rho^{2}} \frac{\mu g}{\mathcal{A}}(N \cdot \Gamma) N .
\end{aligned}
$$

We refer to (7) and its future variations as the disk-polygon system. To apply Theorem 2 we still need to rewrite (7) into the form (6) of an affine system.

Express $v$ in terms of the Frenet frame defined by $T$ and $N: \boldsymbol{v}=\left(v_{T}, v_{N}\right)^{T}$, where $v_{T}=\boldsymbol{v} \cdot T$ and $v_{N}=\boldsymbol{v} \cdot N$. Also express the disk velocity $\boldsymbol{v}_{\mathcal{F}}$ and acceleration $\boldsymbol{a}_{\mathcal{F}}$ in the same frame as $\left(v_{\mathcal{F}_{T}}, v_{\mathcal{F}_{N}}\right)^{T}$ and $\left(a_{\mathcal{F}_{T}}, a_{\mathcal{F}_{N}}\right)^{T}$, respectively. We find that $v_{N}$ depends on $s, \omega$, and $v_{\mathcal{F}_{N}}$ by taking the dot product of $N$ with the velocity constraint (1):

$$
v_{N}=v_{\mathcal{F}_{N}}+s \omega .
$$

System (7) is now rewritten as

$$
\dot{\boldsymbol{x}}=\boldsymbol{f}(\boldsymbol{x})+a_{\mathcal{F}_{T}} \boldsymbol{g}_{T}(x)+a_{\mathcal{F}_{N}} \boldsymbol{g}_{N}(x) .
$$

The state is $\boldsymbol{x}=\left(u, s, \omega, v_{T}, v_{\mathcal{F}_{T}}, v_{\mathcal{F}_{N}}\right)^{T}$; the inputs are the acceleration components $a_{\mathcal{F}_{T}}$ and $a_{\mathcal{F}_{N}}$ along the contact tangent and normal, respectively; and the output is a triple

\footnotetext{
${ }^{2}$ Given a different contact edge $e_{1}$ it follows $\theta=u / r-\pi / 2+\theta_{e_{1}}$ for some constant $\theta_{e_{1}}$.

${ }^{3}$ These equations assume that $O$ and the disk center are on different sides of $e$. Otherwise the term $r+h$ in the following equations for $\dot{s}, \dot{\omega}, \dot{v}$ needs to be replaced by $r-h$.
}

$\boldsymbol{y}=\left(u, v_{\mathcal{F}_{T}}, v_{\mathcal{F}_{N}}\right)^{T}$. The drift and input fields are given as

$$
\boldsymbol{f}(\boldsymbol{x})=\left(\begin{array}{c}
\omega r \\
v_{T}-v_{\mathcal{F}_{T}}-\omega(r+h) \\
\frac{s}{s^{2}+\rho^{2}}\left(\omega^{2}(r+h)\right. \\
\left.-2 \omega\left(v_{T}-v_{\mathcal{F}_{T}}\right)-\frac{\mu g}{\mathcal{A} s} \Gamma_{N}\right) \\
\omega v_{\mathcal{F}_{N}}+s \omega^{2}-\frac{\mu g}{\mathcal{A} s} \Gamma_{T} \\
0 \\
0
\end{array}\right),
$$

$$
\begin{aligned}
& \boldsymbol{g}_{T}(\boldsymbol{x})=(0,0,0,0,1,0)^{T}, \\
& \boldsymbol{g}_{N}(\boldsymbol{x})=\left(0,0,-\frac{s}{s^{2}+\rho^{2}}, 0,0,1\right)^{T} .
\end{aligned}
$$

Theorem 3 The disk-polygon system (8) is locally observable.

Proof By Theorem 2 it suffices to show that the observability codistribution $d \mathcal{O}$ has rank 6 at every state. Now the observation space $\mathcal{O}$ consists of the outputs $u, v_{\mathcal{F}_{T}}, v_{\mathcal{F}_{N}}$ and their repeated Lie derivatives. We choose from $d \mathcal{O}$ the following differentials:

$$
\begin{aligned}
d u & =(1,0,0,0,0,0) ; \\
d v_{\mathcal{F}_{T}} & =(0,0,0,0,1,0) ; \\
d v_{\mathcal{F}_{N}} & =(0,0,0,0,0,1) ; \\
d L_{f} u & =(0,0, r, 0,0,0) ; \\
d L_{g_{N}} L_{f} u & =\left(0, r \frac{s^{2}-\rho^{2}}{\left(s^{2}+\rho^{2}\right)^{2}}, 0,0,0,0\right) ; \\
\left.d L_{g_{N}} L_{f} L_{g_{N}} L_{f} u\right|_{s=\rho} & =\left(0, \frac{r(r+h)}{4 \rho^{4}}, 0,0,0,0\right) .
\end{aligned}
$$

Clearly, it suffices to find one more function in $\mathcal{O}$ whose partial derivative with respect to $v_{T}$ does not vanish.

Such a task is quite easy, for we have

$$
\begin{aligned}
\frac{\partial\left(L_{f} L_{g_{N}} L_{f} u\right)}{\partial v_{T}} & =r \frac{s^{2}-\rho^{2}}{\left(s^{2}+\rho^{2}\right)^{2}} ; \\
\left.\frac{\partial}{\partial v_{T}}\left(L_{f} L_{g_{N}} L_{f} L_{g_{N}} L_{f} u\right)\right|_{s=\rho} & =\frac{r(r+h)}{4 \rho^{4}} .
\end{aligned}
$$

The above proof in fact constructs several control sequences which, when applied for infinitesimal amounts of time, will distinguish between different states in any neighborhood. Assuming $s \neq \rho$, one of the functions $u, v_{\mathcal{F}_{T}}$, $v_{\mathcal{F}_{N}}, L_{f} u, L_{g_{N}} L_{f} u$, and $L_{f} L_{g_{N}} L_{f} u$ must have different values in any two different states close enough as guaranteed by the observability rank condition. Note that $L_{f} u$ is in fact the differential output under zero control. Since 
$L_{g_{N}} L_{f} u$ may be written as $\frac{1}{2} L_{f+g_{N}} L_{f} u-\frac{1}{2} L_{f-g_{N}} L_{f} u$, one of these two functions must distinguish the two states if $L_{g_{N}} L_{f} u$ does. Obviously, $L_{f+g_{N}} L_{f} u$ (or $L_{f-g_{N}} L_{f} u$ ) is realizable in an arbitrarily small amount of time by the control sequence starting with zero control and ending with $a_{\mathcal{F}_{N}}=1$ (or -1$)$. The case with function $L_{f} L_{g_{N}} L_{f} u$ is similar.

Support friction does not affect the local observability of the disk-polygon system, as none of the differentials chosen in the proof to span $d \mathcal{O}$ involve the integral $\Gamma$ or any of its partial derivatives.

\section{Pose Observer}

With the local observability result we can view sensing strategies as nonlinear observers for the disk-polygon system (8) or for the general pushing system (2)-(5). An observer of a nonlinear system is a new system whose state always converges to the state of the original system. The input of the observer consists of the input as well as the output of the original system.

Luenberger-like asymptotic observers [13] for nonlinear systems are often designed through linearization. The diskpolygon system, however, cannot be linearized for we have

$$
L_{g_{N}} L_{f} L_{g_{N}} L_{f} u=r(r+h) \frac{s\left(s^{2}-\rho^{2}\right)}{\left(s^{2}+\rho^{2}\right)^{3}},
$$

violating one of Nijmeijer's necessary conditions [18, p. 156] on linearization. Another approach of observer design transforms the original system into a linear system modulo an output injection [11]. The necessary conditions for a nonlinear system to admit linear observer error dynamics are rather restrictive and hardly satisfied by the disk-polygon system, let alone system (2)-(5).

Our observer, for the disk-polygon system only, uses the following result by Gauthier, Hammouri, and Othman [6].

Theorem 4 (Gauthier, Hammouri, and Othman)

Consider the single output nonlinear (and analytic) system

$$
\begin{aligned}
\dot{\boldsymbol{x}} & =\boldsymbol{f}(\boldsymbol{x}) \\
y & =h(\boldsymbol{x})
\end{aligned}
$$

defined on an $n$-dimensional state space manifold $M$. If

1. the mapping $\chi: x \mapsto \boldsymbol{z}=\left(h(\boldsymbol{x}), \ldots, L_{f}^{n-1} h(\boldsymbol{x})\right)^{T}$ is a diffeomorphism on $M$,

2. $L_{f}^{n} h(\boldsymbol{x})$ can be extended from $M$ to $\Re^{n}$ by a $C^{\infty}$ function that is globally Lipschitzian on $\Re^{n}$,

then the system

$$
\dot{\tilde{\boldsymbol{x}}}=\boldsymbol{f}(\tilde{\boldsymbol{x}})-\frac{\partial \chi^{-1}}{\partial \boldsymbol{z}}(\chi(\tilde{\boldsymbol{x}})) S_{\infty}^{-1} C^{T}(h(\tilde{\boldsymbol{x}})-y)
$$

where $C=(1,0, \ldots, 0)$, and $S_{\infty}$ is the solution of equation

$$
\mathbf{o}=-\zeta S_{\infty}-A^{T} S_{\infty}-S_{\infty} A+C^{T} C,
$$

with $A_{i, j}=\delta_{i, j-1}$, for $\zeta$ large enough, is an observer for (10) with error dynamics

$$
\|\tilde{\boldsymbol{x}}(t)-\boldsymbol{x}(t)\| \leq K(\zeta) e^{-\frac{\zeta t}{3}}\|\tilde{\boldsymbol{x}}(0)-\boldsymbol{x}(0)\| .
$$

The GHO observer for a nonlinear system (6) with inputs is a copy of the original system plus the error corrective term given in (11). To admit such an observer, not only must conditions 1 and 2 in Theorem 4 hold for the drift system $\dot{\boldsymbol{x}}=\boldsymbol{f}(\boldsymbol{x})$, but also the original system must be observable for any input.

Getting back to the disk-polygon system (8), we consider $u, s, \omega$, and $v_{T}$ only as the state variables since $v_{\mathcal{F}_{T}}$ and $v_{\mathcal{F}_{N}}$ are known. The drift and input fields reduce from (9) accordingly. With $u$ being the system's only output, the new coordinates under map $\chi$ consist of $u$ and its Lie derivatives, up to the third order:

$$
\left(\begin{array}{c}
u \\
s \\
\omega \\
v_{T}
\end{array}\right) \quad \stackrel{\chi}{\rightarrow} \quad \boldsymbol{x}=\left(\begin{array}{c}
u \\
\omega r \\
r L_{f} \omega \\
r L_{f}^{2} \omega
\end{array}\right) .
$$

For all except at most a finite number of states, $d u, r d \omega, r d L_{f} \omega$, and $r d L_{f}^{2} \omega$ are linearly independent, so the map $\chi$ is locally diffeomorphic almost everywhere. The Jacobian of the inverse transformation $\chi^{-1}$ is then the inverse of the Jacobian of $\chi$.

Solving equation (12) under $n=4$ for $S_{\infty}$ and plugging it into (11), we obtain an observer for system (8):

$$
\begin{aligned}
\left(\begin{array}{c}
\dot{\tilde{u}} \\
\dot{\tilde{s}} \\
\dot{\tilde{\omega}} \\
\dot{\tilde{v_{T}}}
\end{array}\right) & =f\left(\tilde{u}, \tilde{s}, \tilde{\omega}, \tilde{v_{T}}\right)-\left(\boldsymbol{a}_{\mathcal{F}} \cdot N(\tilde{u})\right) g_{N}(\tilde{s}) \\
& -\left(\begin{array}{cccc}
1 & 0 & 0 & 0 \\
0 & 0 & r & 0 \\
r d L_{f} \omega\left(\tilde{u}, \tilde{s}, \tilde{\omega}, \tilde{v_{T}}\right) \\
r d L_{f}^{2} \omega\left(\tilde{u}, \tilde{s}, \tilde{\omega}, \tilde{v_{T}}\right)
\end{array}\right)\left(\begin{array}{c}
4 \zeta \\
6 \zeta^{2} \\
4 \zeta^{3} \\
\zeta^{4}
\end{array}\right)(\tilde{u}-u) .
\end{aligned}
$$

It should be noted that the second condition in Theorem 4 does not hold since $L_{f}^{4} u$ is generally not extendable to a globally Lipschitzian function. However, $L_{f}^{4} u$ is locally Lipschitzian. The observer we just gave is a local one, like most other nonlinear observers.

\section{Contact Friction}

In the presence of contact friction between the finger and the object, we need to consider two modes of contact: rolling 

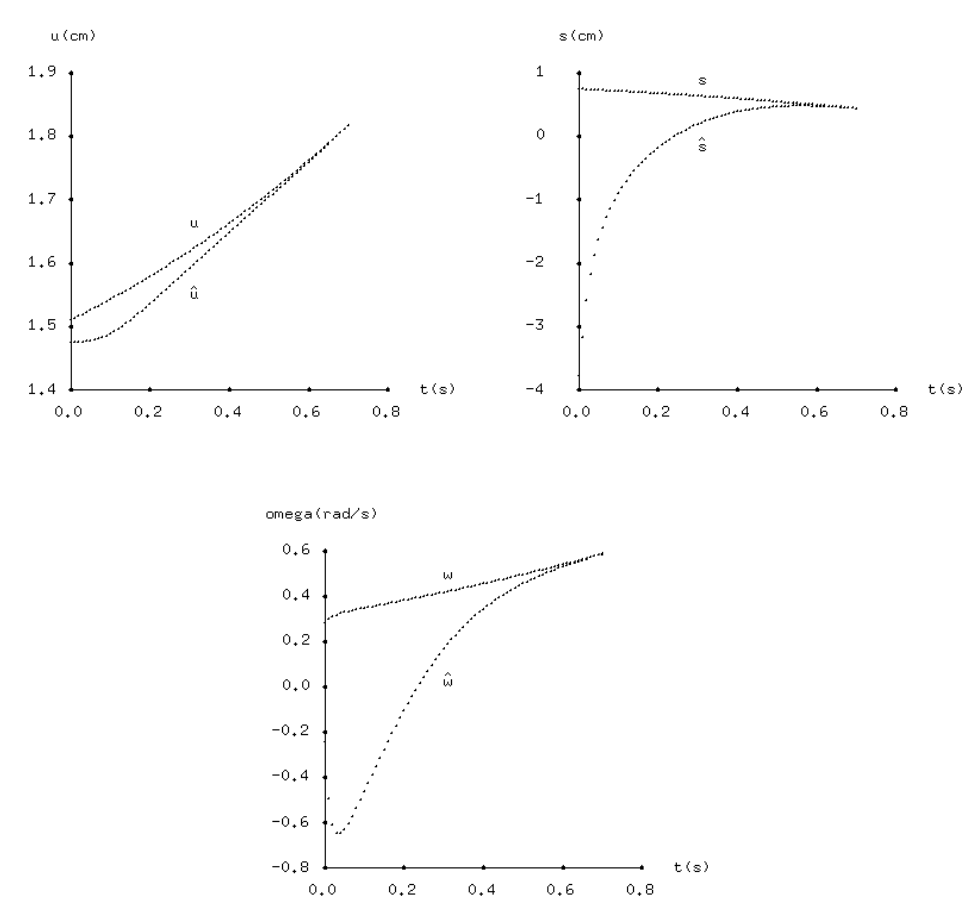

Figure 4: State variable trajectories vs. state estimate trajectories in Figure 1.

and sliding, according to whether the contact force lies inside the contact friction cone or on one of its two edges. Each mode is hypothesized and solved; then the obtained contact force is verified with the contact friction cone for consistency.

It is not difficult to set up motion equations for rolling and sliding that are similar to (2)-(5) and prove that local observability carries over to both situations. A GHO observer for pure rolling can also be derived. We will show the simulation results on this observer in the next section.

\section{Simulations and Experiments}

The GHO observers for frictionless contact (13) and for rolling were simulated. The object data in our simulations were randomly generated polygons. The coefficient of support friction was chosen to be uniformly 0.3. The finger accelerations and velocities used in simulations are achievable on an Adept robot.

For the rolling example shown in Figure 1, the trajectories of $u, s, \omega$ and their estimates $\tilde{u}, \tilde{s}, \tilde{\omega}$ are shown in Figure 4. ${ }^{4}$ Table 1 shows more test results with the same

\footnotetext{
${ }^{4}$ All time measurements in the figure and in the following table refer to the real world not to computer simulation. To give an idea, simulating $1 \mathrm{~s}$ of observation for rolling contact took about 100s, while simulating $1 \mathrm{~s}$
}

polygon and contact edge.

\begin{tabular}{|c|c|c|c|c|c|}
\hline \multirow[t]{2}{*}{ Type } & \multirow{2}{*}{$\begin{array}{c}a_{\mathcal{F}} \\
\left(\mathrm{cm}^{2} / \mathrm{s}\right)\end{array}$} & \multirow[t]{2}{*}{$\zeta$} & \multirow{2}{*}{$\begin{array}{l}\text { No. of } \\
\text { Tests }\end{array}$} & \multicolumn{2}{|c|}{ Successes } \\
\hline & & & & No. & Avg Time (s) \\
\hline Frictionless & 0 & 10 & 30 & 29 & 0.37 \\
\hline Contact & 10 & 10 & 30 & 26 & 0.41 \\
\hline \multirow[t]{4}{*}{ Rolling } & 0 & 10 & 30 & 27 & 0.22 \\
\hline & 0 & 5 & 30 & 25 & 0.56 \\
\hline & 10 & 10 & 30 & 26 & 0.25 \\
\hline & 10 & 5 & 30 & 23 & 0.57 \\
\hline
\end{tabular}

Table 1: Observer performance for the polygon and contact edge in Figure 1. In each test, the real contact $s_{0}$ and its estimate $\tilde{s}_{0}$ were generated randomly on the edge. The disk velocity $v_{\mathcal{F}}$ at the start of observation was always $5 \mathrm{~cm} / \mathrm{s}$ north. The disk acceleration $a_{\mathcal{F}}$ was either $0 \mathrm{~cm} / \mathrm{s}^{2}$, or $10 \mathrm{~cm} / \mathrm{s}^{2}$, which lasted for $0.5 \mathrm{~s}$ before vanishing. The parameter $\zeta$ controls the GHO observers (see Theorem 4). A test was considered a failure if $\tilde{s}$ had not converged to $s$ in $1.5 \mathrm{~s}$.

We have built a "finger" with tactile capability using four strain gauges as shown in Figure 5. The strain gauges are connected to an Omega PC plug-in card to form two Wheatstone half bridges that measure the components of a force exerted on the disk boundary along the $x$ and $y$ axes of the disk, respectively. When contact friction is small enough, the contact force measured by the gauges would be along the disk normal at the contact, thereby indicating the contact point.

The sensor can detect force in microstrains with a frequency over $2000 \mathrm{~Hz}$. It reports a contact with the disk boundary in terms of its polar angle with respect to the disk center. After calibration, the sensed static contacts (in 1000 readings) constantly have a mean within one degree away from the real contact and a standard deviation of less than 0.5 degree.

To realize the GHO observer, we are working on improving the sensor readings of moving contacts which are currently noisy due to varying contact friction.

\section{Summary}

We have introduced a sensing approach based on nonlinear observability theory which makes use of one-finger tactile information. The approach determines the pose of a known planar object by pushing it with a finger that can "feel" the contact motion. It also estimates the object motion during the pushing.

The kinematics of contact and the dynamics of pushing yield a system of nonlinear ODEs whose state includes the object pose and motion and whose output is the moving

of observation for frictionless contact took about $600 \mathrm{~s}$. 


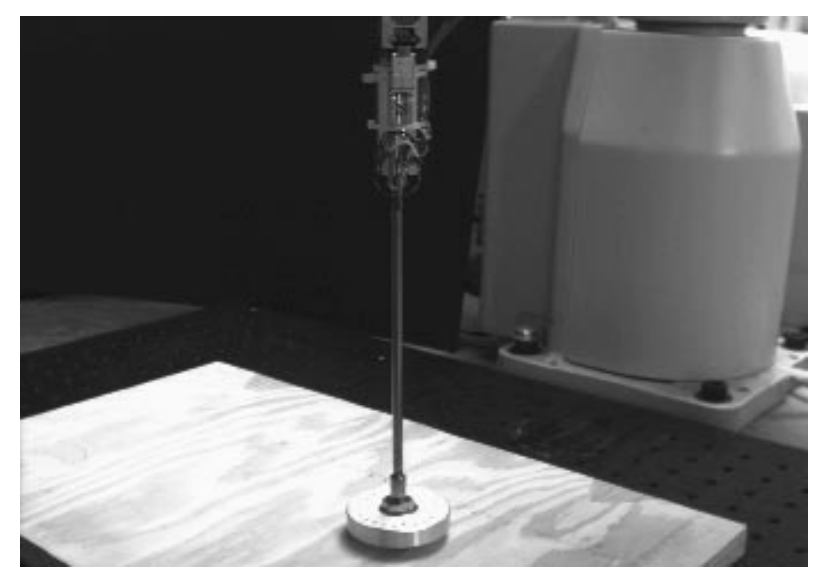

Figure 5: A force sensor for contact sensing. The sensor is composed of a horizontal disk and a cylindrical stainless steel beam erected vertically on the disk and attached to the gripper of an Adept robot at the top. Two pairs of strain gauges are mounted on the upper end of the beam where they would be most sensitive to any force exerted on the disk.

contact on the fingertip. We establish the local observability of this system for the special case of a disk pushing a polygon. Such result is expected to carry over to most other finger and object shapes. This result forms the underlying principle of our sensing algorithm, which is an observer of the nonlinear dynamical system.

Based on the result of [6], we construct asymptotic nonlinear observers taking into account support friction and/or contact friction and demonstrate them by simulations. These online observers are capable of correcting any local error in estimating the object pose and motion.

We have implemented a force sensor using strain gauges to detect contact locations. Preliminary experiments have been carried out with an Adept robot.

Our undergoing research focuses on the sensor experiments and the extension of pose and motion observability to 3-D tasks.

Acknowledgements Thanks to Matt Mason, Kevin Lynch, Wes Huang, and Alfred Rizzi for their insights and discussions on force sensing, pushing, and nonlinear observers; to Ken Salisbury, Alan Guisewite, and Ralph Hollis for their feedback on the experimental setup, and especially, on the use of strain gauges; to Garth Zeglin for generous help with the experiments. We also thank the anonymous reviewers for their detailed and insightful comments.

\section{References}

[1] J. C. Alexander and J. H. Maddocks. Bounds on the frictiondominated motion of a pushed object. Int. J. Robot. Res., 12(3):231-248, 1993.
[2] P. K. Allen and K. S. Roberts. Haptic object recognition using a multi-fingered dextrous hand. In Proc. 1989 IEEE Int. Conf. Robotics and Automation, pages 342-347, 1989.

[3] C. Cai and B. Roth. On the spatial motion of a rigid body with point contact. In Proc. 1987 IEEE Int. Conf. Robotics and Automation, pages 686-695, 1987.

[4] G. Ciccarella, M. D. Mora, and A. Germani. A Luenbergerlike observer for nonlinear systems. Int. J. Control, 57(3):537-556, 1993.

[5] R. S. Fearing and T. O. Binford. Using a cylindrical tactile sensor for determining curvature. In Proc. 1988 IEEE Int. Conf. Robotics and Automation, pages 765-771, 1988.

[6] J. P. Gauthier, H. Hammouri, and S. Othman. A simple observer for nonlinear systems applications to bioreactors. IEEE Trans. Auto. Control, 37(6):875-880, 1992.

[7] S. Goyal, A. Ruina, and J. Papadopoulos. Planar sliding with dry friction. Wear, 143:307-352, 1991.

[8] R. Hermann and A. J. Krener. Nonlinear controllability and observability. IEEE Trans. Auto. Control, AC-22(5):728$740,1977$.

[9] A. Isidori. Nonlinear Control Systems: An Introduction. Springer-Verlag, 1985.

[10] Y.-B. Jia and M. Erdmann. Pose from pushing. In Proc. 1996 IEEE Int. Conf. Robotics and Automation, pages 165-171, 1996.

[11] A. J. Krener and A. Isidori. Linearization by output injection and nonlinear observers. Sys. Control Letters, 3:47-52, 1983.

[12] A. J. Krener and W. Respondek. Nonlinear observers with linearizable error dynamics. SIAM J. Control and Optimization, 23(2):197-216, 1985.

[13] D. G. Luenberger. An introduction to observers. IEEE Trans. Auto. Control, AC-16(6), 1971.

[14] K. Lynch, H. Maekawa, and K. Tanie. Manipulation and active sensing by pushing using tactile feedback. In Proc. 1992 IEEE/RSJ Int. Conf. Intell. Robots and Systems, pages 416421, 1992.

[15] W. D. MacMillan. Dynamics of Rigid Bodies. McGraw-Hill Book Company Inc., 1936. Dover reprint, 1960.

[16] M. T. Mason. Mechanics and planning of manipulator pushing operations. Int. J. Robot. Research, 5(3):53-71, 1986.

[17] D. J. Montana. The kinematics of contact and grasp. Int. J. Robot. Research, 7(3):17-32, 1988.

[18] H. Nijmeijer and A. van der Schaft. Nonlinear Dynamical Control Systems. Springer-Verlag, New York, 1990.

[19] K. Salisbury. Interpretation of contact geometries from force measurements. In M. Brady and R. Paul, editors, Robotics Research, pages 565-577. The MIT Press, 1984.

[20] G. Zimmer. State observation by on-line minimization. Int. J. Control, 60(4):595-606, 1994. 